\title{
Subtle hemorrhagic brain injury is associated with neurodevelopmental impairment in infants with repaired congenital heart disease
}

\author{
Janet S. Soul, MD, CM, ${ }^{\mathrm{a}, \mathrm{f}}$ Richard L. Robertson, MD, ${ }^{\mathrm{b}, \mathrm{g}}$ David Wypij, PhD, ${ }^{\text {,h,k }}$ David C. Bellinger, PhD, \\ MSc ${ }^{\text {a,f }}$ Karen J. Visconti, PhD, ${ }^{\text {e }}$ Adré J. du Plessis, MBChB, MPH, ${ }^{\text {a,f }}$ Barry D. Kussman, MBBCh, d,i \\ Lisa A. Scoppettuolo, MS, ${ }^{\mathrm{c}, \mathrm{k}}$ Frank Pigula, MD, ${ }^{\mathrm{e}, \mathrm{j}}$ Richard A. Jonas, MD, ${ }^{\mathrm{e}, \mathrm{j}}$ and Jane W. Newburger, MD, \\ $\mathrm{MPH}^{\mathrm{c}, \mathrm{h}}$
}

\begin{abstract}
Objective: Perioperative stroke and periventricular leukomalacia have been reported to occur commonly in infants with congenital heart disease. We aimed to determine the incidence and type of brain injury in infants undergoing 2-ventricle repair in infancy and to determine risk factors associated with such injury.

Methods: Forty-eight infants enrolled in a trial comparing 2 different hematocrits during surgical repair of congenital heart disease underwent brain magnetic resonance imaging scans and neurodevelopmental testing at 1 year of age.
\end{abstract}

Results: Eighteen (38\%) of our subjects had tiny foci of hemosiderin by susceptibility imaging, without evidence of abnormalities in corresponding regions on conventional magnetic resonance imaging sequences. Subjects with foci of hemosiderin had a significantly lower Psychomotor Developmental Index at 1 year of age $(79.6 \pm 16.5$, mean \pm standard deviation) compared with subjects without these foci $(89.5 \pm 15.3 ; P=.04)$. Older age at surgery and diagnostic group were significantly associated with the presence of hemosiderin foci. Only 1 subject had a small stroke $(2 \%)$, and 2 subjects had periventricular leukomalacia $(4 \%)$.

Conclusion: Foci of hemosiderin without radiologic evidence of ischemic brain injury are an abnormality associated with adverse neurodevelopmental outcome not previously described in magnetic resonance imaging studies of children with surgically repaired congenital heart disease. The association of hemosiderin foci with older age at surgery and cardiac diagnosis, and not with risk factors associated with brain injury, in previous studies suggests that the cause and pathogenesis of this abnormality are different from ischemic brain lesions reported previously.

Despite advances in the care of children with congenital heart disease (CHD), neurodevelopmental impairments remain common in this population. ${ }^{1-9}$ Deficits may occur in cognition and behavior, as well as in motor domains. ${ }^{5-8}$ However, the relationship of these neurodevelopmental impairments with specific neuropathologic abnormalities remains unclear. One recent neuropathologic study of children who died days to months after early surgical repair of CHD

From the Departments of Neurology, ${ }^{\mathrm{a}}$ Radiology, ${ }^{\mathrm{b}}$ Cardiology, ${ }^{\mathrm{c}}$ Anesthesiology, ${ }^{\mathrm{d}}$ and Cardiovascular Surgery, at Children's Hospital Boston; the Departments of Neurology ${ }^{\mathrm{f}}$ Radiology, ${ }^{\mathrm{g}}$ Pediatrics, ${ }^{\mathrm{h}}$ Anesthesia, ${ }^{\mathrm{i}}$ and Surgery, ${ }^{\mathrm{j}}$ at Harvard Medical School, and the Department of Biostatistics, ${ }^{k}$ Harvard School of Public Health, Boston, Mass.

Supported by grants HL 063411 and RR 02172 from the National Institutes of Health. Registered with clinicaltrials.gov (\#) NCT00006183.

Dr Jonas is currently Chief of Cardiovascular Surgery and Co-Director of the Children's National Heart Institute, Washington, DC.

Received for publication Aug 1, 2008; revisions received Nov 13, 2008; accepted for publication Feb 9, 2009; available ahead of print April 13, 2009.

Address for reprints: Janet S. Soul, MD, CM, Department of Neurology, Children's Hospital Boston, 300 Longwood Avenue, Boston, MA 02115 (E-mail: janet. soul@childrens.harvard.edu).

J Thorac Cardiovasc Surg 2009; 138:374-81

$0022-5223 / \$ 36.00$

Copyright (c) 2009 by The American Association for Thoracic Surgery

doi:10.1016/j.jtcvs.2009.02.027 showed that diffuse white matter gliosis and periventricular leukomalacia (PVL) were the dominant lesions. ${ }^{10}$ In MRI studies of children surviving CHD, several groups have reported a relatively high incidence of PVL and focal stroke. ${ }^{11-14}$ However, no reports have demonstrated a direct correlation between PVL or stroke and subsequent neurodevelopmental deficits.

By contrast, numerous studies have delineated perioperative factors that may contribute to the pathogenesis of brain injury that likely underlies later neurodevelopmental deficits. ${ }^{1,3,5,6,9,15-19}$ For example, previous reports have shown that balloon atrial septostomy (BAS), lower Apgar score at 5 minutes, lower intraoperative cerebral oxygenation saturation, and lower blood pressure in the postoperative period were risk factors associated with radiologically apparent brain injury, specifically PVL and stroke. ${ }^{12-14}$ A previous trial conducted at Children's Hospital Boston showed a significant effect of hematocrit during bypass surgery on neurologic and cardiac outcomes. ${ }^{16}$ In that study, a higher hematocrit was associated with improved cardiac and neurologic outcomes. ${ }^{16}$ In a subsequent randomized trial comparing 2 higher hematocrits $(35 \%$ vs $25 \%$ ), the treatment groups were similar with respect to neurologic outcomes 


\section{Abbreviations and Acronyms \\ BAS = balloon atrial septostomy \\ BSID-II = Bayley Scales of Infant Development II \\ CHD = congenital heart disease \\ MDI = Mental Development Index \\ MPGR = multiplanar gradient recalled \\ PDI = Psychomotor Development Index \\ PVL = periventricular leukomalacia \\ TGA $=$ D-transposition of the great arteries \\ TOF $=$ tetralogy of Fallot \\ VSD $=$ ventricular septal defect}

measured by brain MRI and neurodevelopmental evaluation at 1 year of age. ${ }^{20}$

In a substudy of the latter hematocrit trial, we sought to determine the incidence of radiologically apparent brain injury by brain MRI at 1 year of age, the perioperative factors associated with brain injury, and the association of brain injury with 1-year neurodevelopmental outcomes. We hypothesized that BAS, hypotension, and longer bypass, circulatory arrest, or crossclamp times would be associated with a higher incidence of radiologically apparent brain injury, and that brain injury found by MRI scan would be associated with worse neurodevelopmental outcome.

\section{MATERIALS AND METHODS}

We enrolled infants less than 9 months of age requiring surgical repair of 1 of the 3 following congenital heart defects: (1) D-transposition of the great arteries (TGA) with intact ventricular septum or ventricular septal defect (VSD); (2) tetralogy of Fallot (TOF) with or without pulmonary atresia or truncus arteriosus; and (3) VSD or complete common atrioventricular canal defect. Infants were excluded if they had birth weight less than $2.3 \mathrm{~kg}$, recognizable phenotypic syndrome of congenital anomalies, extracardiac anomalies of greater than minor severity, previous cardiac surgery, or associated cardiovascular anomalies requiring aortic arch reconstruction or additional open surgical procedures before the planned developmental follow-up. Infants were randomized to a hematocrit strategy of either $25 \%$ or $35 \%$, with stratification by surgeon and diagnostic group. A detailed description of the study design, anesthesia/perfusion methods, and study outcomes has been reported. ${ }^{20}$ We obtained written informed consent in all cases. The study was approved by the institutional review board at Children's Hospital Boston. We collected relevant clinical variables regarding the subjects' preoperative, intraoperative, and postoperative courses, and 1-year neurologic and developmental evaluations as described previously. Specifically, the Mental Development Index (MDI) and Psychomotor Development Index (PDI) were obtained for each subject at 1 year of age using the Bayley Scales of Infant Development II (BSID-II).

We obtained brain MRI data at 1 year of age in a subset of subjects enrolled in the trial. We obtained a sagittal localizer, a standard clinical axial fast spin echo T2-weighted sequence (TE 84, TR 4000, flip angle 90), and an axial multiplanar gradient recalled (MPGR) acquisition susceptibility weighted sequence (TE 40, TR 600, flip angle 30) for the detection of prior hemorrhage. Three-dimensional SPoiled Gradient Recalled (with TE 5, TR 35) and dual echo fast spin echo sequences (TE 50 and effective TE 150, TR 5000 ) were acquired in the coronal plane. We obtained axial diffusion tensor imaging data ${ }^{21}$ with 6 diffusion directions and $b$ factors of 5 and $750 \mathrm{~s} / \mathrm{mm}^{2}$. Finally, we obtained single voxel proton magnetic resonance spectroscopy using a point-resolved spectroscopy sequence technique (TR $1500 \mathrm{~ms}$, TE $144 \mathrm{~ms}$ ) with the voxel placed in the left parieto-occipital cerebrum (largely white matter), lateral and superior to the trigone of the lateral ventricle.

All MRI sequences were evaluated for evidence of acquired or developmental abnormalities by a single pediatric neuroradiologist (R.L.R.) who was unaware of the cardiac diagnosis, medical history, or perioperative course of the subjects. The images were evaluated prospectively in a standardized fashion, and abnormalities were recorded using a form developed specifically for this trial. The images were examined for the presence of focal or multifocal acquired abnormalities, such as focal stroke, hemorrhage, atrophy, or mineralization, and diffuse abnormalities, including ventriculomegaly, delayed myelination, or diffuse $\mathrm{T} 2$ prolongation indicative of white matter gliosis. Imaging was also reviewed for the presence of developmental abnormalities, including major anomalies such as schizencephaly or agenesis of the corpus callosum, or minor developmental abnormalities such as a persistent cavum septum pellucidum, Chiari I malformation, or arachnoid cyst.

Categoric perioperative factors and MRI abnormalities were compared across the 3 cardiac diagnostic groups using Fisher's exact test. Continuous perioperative factors were compared across diagnostic groups using Kruskal-Wallis tests. Developmental outcomes were compared across diagnostic groups using analysis of variance. The relationships between perioperative characteristics reported in Table 1 and MRI abnormalities were investigated using logistic regression. The relationship between MRI abnormalities and developmental outcome measures (ie, MDI and PDI) was investigated using $t$ tests and linear regression, adjusting for diagnostic group. Statistical analyses were performed using SAS Version 9.1 (SAS Institute Inc, Cary, NC), and statistical significance was set at the .05 level.

\section{RESULTS}

MRI scans were obtained in 48 of 106 subjects who returned for 1-year follow-up evaluations $(n=25$ and $n=23$ in the $25 \%$ and $35 \%$ hematocrit groups, respectively). The mean age at MRI was 1.2 years ( standard deviation $=0.2$ ). In the remaining subjects, MRI scans were not obtained because of parental refusal, usually related to the need for general anesthesia ( 55 subjects, $52 \%$ ), cancellations by the anesthesiologist because of medical concerns $(2,2 \%)$, and no MRI machine available $(1,1 \%)$. Among the subjects who returned for the 1-year evaluation, those who underwent brain MRI, compared with those who did not, were similar with respect to scores on the BSID-II and neurologic examination.

\section{Clinical Characteristics of Subjects}

Clinical perioperative characteristics of subjects who underwent a brain MRI scan, according to diagnostic group, are shown in Table 1. Boys outnumbered girls in the TGA (12 vs 7) and VSD (6 vs 3 ) groups, but the cardiac diagnostic groups were similar with respect to birth weight, gestational age at birth, Apgar scores, and hematocrit at onset of lowflow bypass (Table 1). Diagnostic group was highly associated with age at surgery. Subjects with TGA underwent neonatal surgical repair, whereas subjects with TOF and VSD generally underwent surgery at 2 to 5 months of age. Of the 19 subjects with TGA, 17 had BAS before surgery (3 BASs performed in the catheterization laboratory and 14 BASs performed at the bedside) and 2 had no BAS. Only 2 subjects received a bolus of heparin before the BAS. No 
TABLE 1. Characteristics of 48 subjects undergoing infant heart surgery who had magnetic resonance imaging at 1 year, according to diagnosis group

\begin{tabular}{|c|c|c|c|c|c|}
\hline Variable & $\operatorname{ALL}(\mathbf{N}=48)$ & TGA $(\mathbf{N}=19)$ & TOF $(N=20)$ & $\operatorname{VSD}(\mathbf{N}=9)$ & $P$ value* \\
\hline \multicolumn{6}{|l|}{ Preoperative characteristics } \\
\hline Birth weight, mean $\pm \mathrm{SD}$ (kg) & $3.4 \pm 0.5$ & $3.6 \pm 0.6$ & $3.3 \pm 0.5$ & $3.4 \pm 0.5$ & .32 \\
\hline Gestational age, mean $\pm \mathrm{SD}$ (wk) & $39.2 \pm 1.3$ & $39.0 \pm 1.2$ & $39.3 \pm 1.1$ & $39.2 \pm 1.8$ & .56 \\
\hline Apgar score at 5 min, mean $\pm \mathrm{SD}$ & $8.5 \pm 1.2$ & $8.2 \pm 1.7$ & $8.7 \pm 0.7$ & $8.9 \pm 0.3$ & .24 \\
\hline Sex, $\%$ male & 58 & 63 & 50 & 67 & .64 \\
\hline Race, \% nonwhite & 21 & 16 & 15 & 44 & .19 \\
\hline Age at surgery, median (range) (d) & $41(2-264)$ & $6(2-13)$ & $63(2-198)$ & $156(39-264)$ & $<.001$ \\
\hline Intubated before surgery, $\dagger \%$ & 10 & 21 & 5 & 0 & .19 \\
\hline Abnormal neurologic examination, $\mathrm{n} /$ total $(\%)$ & $17 / 36(47)$ & $8 / 15(53)$ & $5 / 13(38)$ & $4 / 8(50)$ & .70 \\
\hline \multicolumn{6}{|l|}{ Operative characteristics, mean \pm SD } \\
\hline Crossclamp time (min) & $64 \pm 26$ & $90 \pm 21$ & $49 \pm 10$ & $44 \pm 11$ & $<.001$ \\
\hline Total support time (min) & $103 \pm 35$ & $140 \pm 25$ & $82 \pm 11$ & $72 \pm 13$ & $<.001$ \\
\hline Total bypass time (min) & $96 \pm 30$ & $124 \pm 26$ & $80 \pm 12$ & $72 \pm 13$ & $<.001$ \\
\hline Low-flow bypass time (min) & $48 \pm 26$ & $70 \pm 24$ & $33 \pm 16$ & $33 \pm 16$ & $<.001$ \\
\hline Duration of circulatory arrest (min) & $7 \pm 11$ & $16 \pm 11$ & $3 \pm 7$ & $0 \pm 0$ & $<.001$ \\
\hline Hematocrit at onset of low-flow bypass ( $\%)$ & $28.5 \pm 5.3$ & $28.0 \pm 5.2$ & $28.0 \pm 5.4$ & $30.4 \pm 5.9$ & .47 \\
\hline Nadir temperature $\left({ }^{\circ} \mathrm{C}\right)$ & $20.0 \pm 5.6$ & $15.2 \pm 1.4$ & $21.7 \pm 5.4$ & $26.3 \pm 1.7$ & $<.001$ \\
\hline Lowest $\mathrm{PCO}_{2}(\mathrm{~mm} \mathrm{Hg})$ & $32.6 \pm 5.1$ & $32.6 \pm 5.1$ & $32.5 \pm 5.0$ & $33.0 \pm 5.9$ & .98 \\
\hline Highest $\mathrm{PCO}_{2}(\mathrm{~mm} \mathrm{Hg})$ & $76.0 \pm 15.0$ & $86.0 \pm 12.4$ & $71.0 \pm 14.5$ & $65.9 \pm 8.6$ & $<.001$ \\
\hline Lowest $\mathrm{PO}_{2}(\mathrm{~mm} \mathrm{Hg})$ & $90.3 \pm 80.4$ & $47.8 \pm 13.1$ & $103.1 \pm 95.9$ & $151.7 \pm 82.4$ & $<.001$ \\
\hline \multicolumn{6}{|l|}{ Postoperative variables } \\
\hline Lactate 60 min after bypass, mean $\pm \mathrm{SD}$ & $2.7 \pm 1.5$ & $3.9 \pm 1.5$ & $2.1 \pm 0.9$ & $1.7 \pm 0.7$ & $<.001$ \\
\hline \multicolumn{6}{|l|}{ PRISM III score, mean \pm SD } \\
\hline $12 \mathrm{~h}$ postoperatively & $5.5 \pm 3.3$ & $6.2 \pm 2.2$ & $6.2 \pm 3.8$ & $2.3 \pm 1.8$ & .001 \\
\hline $24 \mathrm{~h}$ postoperatively & $7.4 \pm 3.8$ & $8.1 \pm 2.5$ & $8.5 \pm 4.5$ & $3.7 \pm 2.2$ & .002 \\
\hline Lowest $\mathrm{PaCO}_{2}$, mean $\pm \mathrm{SD}$ & $33.6 \pm 4.5$ & $31.0 \pm 3.9$ & $34.1 \pm 4.1$ & $37.5 \pm 3.7$ & .002 \\
\hline Lowest $\mathrm{PO}_{2}$, mean $\pm \mathrm{SD}$ & $75.9 \pm 23.9$ & $88.4 \pm 23.2$ & $63.8 \pm 20.9$ & $78.0 \pm 19.8$ & .003 \\
\hline Days intubated, median (interquartile range) & $1.7(1.1-2.9)$ & $2.0(1.2-3.6)$ & $2.0(1.2-2.9)$ & $1.0(0.8-1.1)$ & .002 \\
\hline \multicolumn{6}{|l|}{ Follow-up data at $1 \mathrm{y}$} \\
\hline Abnormal neurologic examination, $\mathrm{n} /$ total $(\%)$ & $28 / 48(58)$ & $7 / 19(37)$ & $14 / 20(70)$ & $7 / 9(78)$ & .06 \\
\hline $\mathrm{PDI}$, mean $\pm \mathrm{SD}$ & $85.8 \pm 16.3$ & $84.8 \pm 14.9$ & $86.5 \pm 19.7$ & $86.3 \pm 11.9$ & .95 \\
\hline MDI, mean \pm SD & $94.7 \pm 11.3$ & $96.6 \pm 10.2$ & $93.9 \pm 13.3$ & $92.3 \pm 8.5$ & .61 \\
\hline
\end{tabular}

TGA, D-transposition of the great arteries; TOF, tetralogy of Fallot; VSD, ventral septal defect; PDI, Psychomotor Developmental Index; $M D I$, Mental Developmental Index; $S D$, standard deviation; $\mathrm{PCO}_{2}$, carbon dioxide tension; $\mathrm{PO}_{2}$, oxygen tension. ${ }^{*} P$ values are for differences between diagnostic groups. For dichotomous variables, $P$ values were determined by exact tests. For PDI and MDI, $P$ values were determined by analysis of variance. For all other continuous variables, $P$ values were determined by Kruskal-Wallis tests. $\dagger$ Excludes patients who were transiently intubated before surgery.

subject underwent a postoperative catheterization before the MRI scan and neurodevelopmental testing at 1 year of age.

The diagnostic groups differed in most intraoperative and postoperative variables (Table 1). Infants in the TGA group had the longest operations, greatest use of total circulatory arrest, lowest minimum temperature, and greatest postoperative morbidity as indicated by highest lactate 60 minutes after bypass, 12 and 24-hour PRISM III scores, and number of days of endotracheal intubation. Conversely, infants in the VSD group had the lowest intraoperative and postoperative complexity. No subjects had postoperative hypotension, defined as a systolic blood pressure less than $40 \mathrm{~mm} \mathrm{Hg}$ in a neonate or less than $45 \mathrm{~mm} \mathrm{Hg}$ in an infant more than 30 days old.

The percentage of children with abnormal neurologic examination results at age 1 year tended to differ among the 3 diagnostic groups $(P=.06$, Table 1$)$. Despite the greater peri- operative morbidity of children with TGA, their prevalence of abnormal neurologic examination results $(37 \%)$ was lower than that of infants in the TOF and VSD groups $(70 \%$ and $78 \%$, respectively). The diagnostic groups did not differ significantly in their scores on the PDI or MDI of the BSID-II.

\section{Magnetic Resonance Imaging Data}

Qualitative analysis of the image data showed no evidence of severe brain injury or major brain malformations (Table 2). We found more acquired or developmental abnormalities in subjects with VSD (7 abnormal vs 2 normal), compared with the TOF (11 vs 9) or TGA (5 vs 14$)$ groups $(P=.03)$. The majority of the acquired abnormalities were tiny foci of signal abnormality on the MPGR sequence consistent with small foci of hemosiderin in 18 subjects $(38 \%$, Figure 1). The foci were found throughout the cerebral 
TABLE 2. Qualitative evaluation of magnetic resonance imaging outcomes according to diagnosis group

\begin{tabular}{|c|c|c|c|c|c|}
\hline Variable & $\operatorname{ALL}(\mathbf{N}=\mathbf{4 8})$ & TGA $(\mathbf{N}=19)$ & TOF $(\mathbf{N}=20)$ & $\operatorname{VSD}(\mathbf{N}=\mathbf{9})$ & $P$ value* \\
\hline \multirow[t]{2}{*}{ Age at MRI, mean $\pm \mathrm{SD}(\mathrm{y})$} & $1.2 \pm 0.2$ & $1.1 \pm 0.1$ & $1.2 \pm 0.2$ & $1.3 \pm 0.3$ & .10 \\
\hline & \multicolumn{5}{|c|}{ No. with abnormality (\%) } \\
\hline Any acquired or developmental abnormalities & $23(48)$ & $5(26)$ & $11(55)$ & $7(78)$ & .03 \\
\hline Focal or multifocal abnormalities & $19(40)$ & $4(21)$ & $9(45)$ & $6(67)$ & .06 \\
\hline Focal infarction or atrophy & $1(2)$ & $1(5)$ & 0 & 0 & .58 \\
\hline Brain mineralization/hemosiderin & $18(38)$ & $3(16)$ & $9(45)$ & $6(67)$ & .03 \\
\hline Diffuse abnormalities & $3(6)$ & $1(5)$ & $1(5)$ & $1(11)$ & .79 \\
\hline Delayed myelination & $1(2)$ & 0 & 0 & $1(11)$ & .19 \\
\hline Ventriculomegaly & $1(2)$ & $1(5)$ & 0 & 0 & .58 \\
\hline T2 hyperintensities/gliosis/periventricular leukomalacia & $2(4)$ & 0 & $1(5)$ & $1(11)$ & .49 \\
\hline Developmental abnormalities & $4(8)$ & 0 & $3(15)$ & $1(11)$ & .23 \\
\hline Major malformation & 0 & 0 & 0 & 0 & 1.0 \\
\hline Minor malformation & $4(8)$ & 0 & $3(15)$ & $1(11)$ & .23 \\
\hline
\end{tabular}

$T G A$, D-transposition of the great arteries; $T O F$, tetralogy of Fallot; $V S D$, ventral septal defect; $M R I$, magnetic resonance imaging; $S D$, standard deviation. $* P$ values are for differences among diagnostic groups. For age at MRI, the $P$ value was determined by a Kruskal-Wallis test. For dichotomous variables, $P$ values were determined by exact tests.

cortex and white matter, basal ganglia, and cerebellum, without predilection for any particular brain region or any specific pattern of distribution. Nine subjects had a single focus of hemosiderin, and the other 9 subjects had 2 or more such foci. No signal abnormality was found in the region of these foci on any of the corresponding $\mathrm{T} 1 \mathrm{w}$ or $\mathrm{T} 2 \mathrm{w}$ images (Figure 1).

In addition to the hemorrhagic foci, several other abnormalities were present. One female subject with TGA had a small area of focal encephalomalacia in the left insular region consistent with a small focal stroke (Figure 2), but focal stroke was not seen in any other subjects (incidence of $2 \%$ ). The subject with focal stroke underwent a preoperative BAS in the catheterization laboratory without heparin; thus, the incidence of focal stroke among infants with TGA undergoing BAS and surgical repair was 1 of $17(6 \%)$. PVL was found only in 2 subjects (4\%): 1 female subject with VSD and 1 male subject with TOF (Figure 3). The subject with VSD and PVL was judged to have globally delayed myelination (Figure 3, $A, B$ ). One female subject with TGA had ventriculomegaly but did not have evidence of any white matter or other parenchymal abnormality. We detected minor developmental abnormalities in 4 subjects, 3 of whom had a Chiari I malformation and 1 of whom had prominent perivascular spaces in the periatrial white matter. We found no major developmental abnormalities in our subjects.

\section{Relationship Between Magnetic Resonance Imaging Abnormalities and Neurodevelopmental Outcome}

Subjects with foci of hemosiderin, compared with those without such foci, had a 10-point lower mean PDI score (79.6 \pm 16.5 vs $89.5 \pm 15.3$, respectively; $t$ test $P=.04)$ but were similar with respect to MDI scores (Figure 4). The association of hemosiderin with lower mean PDI score remained statistically significant in linear regression when adjusted for cardiac diagnostic group $(P=.02)$. Although no other MRI abnormalities were associated with developmental outcome, our power to detect differences was low given the low frequency of each of the other abnormalities, for example, only 2 cases of PVL.

\section{Relationship Between Perioperative Characteristics and Magnetic Resonance Imaging Abnormalities}

We explored whether perioperative variables were associated with the presence of foci of hemosiderin. Eleven subjects received antifibrinolytic medications, including aprotinin ( 8 subjects), tranexamic acid (2 subjects), and aminocaproic acid (1 subject). There were no significant associations between antifibrinolytics administered (individually or combined) and the presence of hemosiderin $(P=1.0$ for all). Cardiac diagnosis was the main characteristic associated with the presence of foci of hemosiderin. These foci were found in only 3 of 19 subjects with TGA (16\%), but in 9 of 20 subjects with TOF (45\%) and 6 of 9 subjects with $\operatorname{VSD}(67 \% ; P=.03$ comparing the 3 groups). Second, we found that older age at surgery, both as a continuous variable and as a categoric variable ( $>30$ days vs $\leq 30$ days), was associated with a higher rate of hemosiderin. Specifically, hemosiderin was detected in 15 of 26 subjects $(58 \%)$ ) whose age at surgery was more than 30 days versus 3 of 22 subjects $(14 \%)$ whose age at surgery was 30 days or less (Fisher's exact test, $P=.003$ ). Because age at surgery was closely related to cardiac diagnosis, it was not possible to determine whether cardiac diagnosis (including conduct of surgical repair) or age at surgery was the main factor associated with hemosiderin. We performed logistic regression within each cardiac diagnosis group with age at surgery as a continuous or dichotomized ( $\leq />30$ days) predictor and did not find any statistically significant correlations, although the small numbers within each diagnosis group limited the power of these analyses. In additional univariate analyses, we found that higher nadir of intraoperative temperature was associated with the 

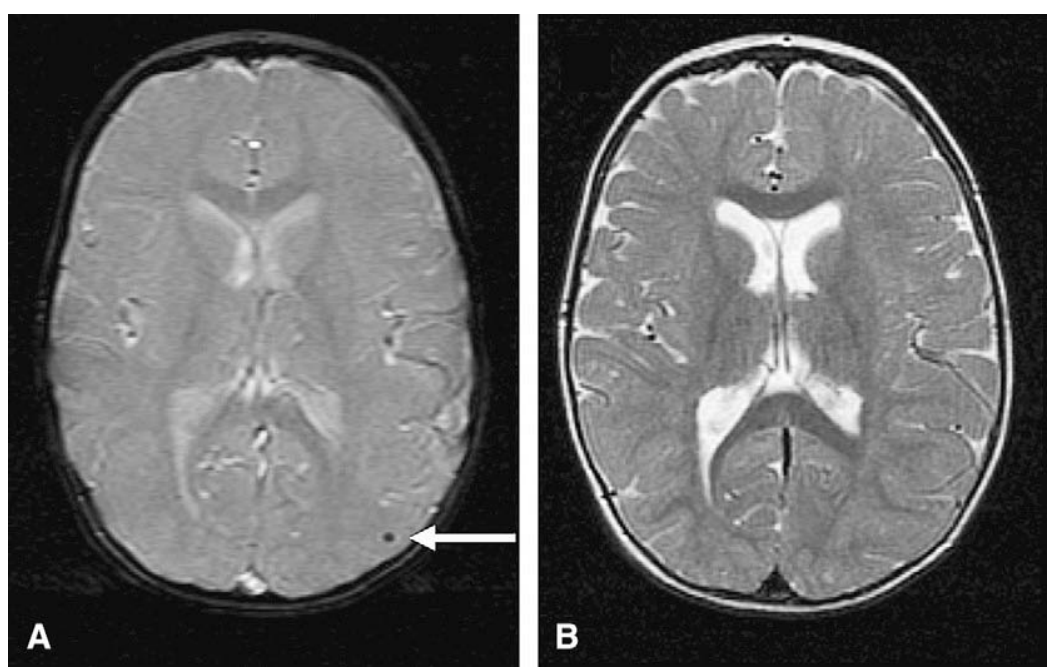

FIGURE 1. Focus of hemosiderin in left parieto-occipital cortex (arrow) seen on axial MPGR sequence (A) without corresponding abnormality on axial T2 $\mathrm{w}$ fast spin echo sequence (B).

presence of hemosiderin $(P=.003)$, as was higher nadir of postoperative $\mathrm{PaCO}_{2}(P=.008)$. However, when the relationship between hemosiderin and either lowest intraoperative temperature or lowest postoperative $\mathrm{PaCO}_{2}$ was adjusted for age at surgery, the associations were no longer statistically significant. The strong association among variables, including lowest intraoperative temperature and postoperative $\mathrm{PaCO}_{2}$, cardiac diagnosis, and age at surgery, limited our ability to determine which factors were explanatory. No other perioperative clinical variables, such as abnormal findings

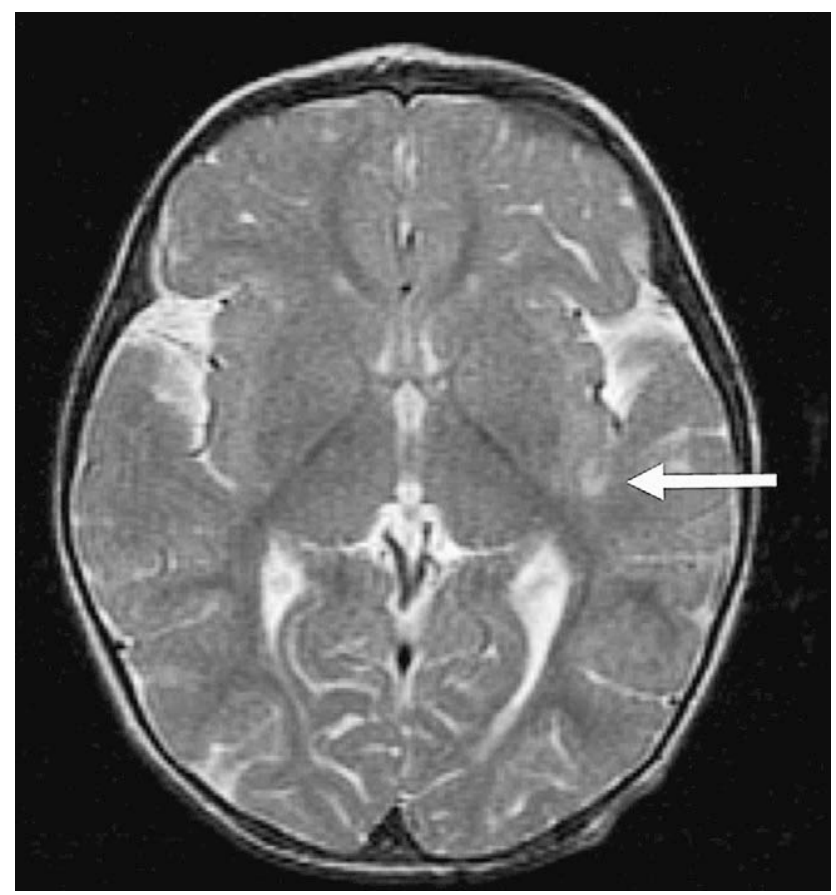

FIGURE 2. Small area of focal encephalomalacia seen as T2 hyperintensity in left insular region (arrow), consistent with old focal stroke. on preoperative neurologic examination, were significantly associated with the presence of hemosiderin. Finally, the number of subjects with focal stroke $(n=1)$ and PVL $(n=2)$ was inadequate to analyze which perioperative variables were associated with these brain lesions.

\section{DISCUSSION}

This study demonstrates a new finding of foci of signal loss on the susceptibility-weighted MRI sequence in a population of children undergoing 2-ventricle repair in infancy. Specifically, $38 \%$ of our subjects had tiny foci of magnetic susceptibility artifact thought to be hemosiderin scattered throughout the cerebral and cerebellar parenchyma. This finding has not been reported in previous MRI studies of infants undergoing surgical repair of CHD in which a susceptibility weighted sequence was performed. ${ }^{11,12,22}$ The presence of these foci was associated with significantly lower PDI score at 1 year of age in our subjects, suggesting that this is a clinically important finding.

The neuropathology associated with the foci of susceptibility artifact seen in our patients is currently unknown. Susceptibility-weighted imaging is a gradient-echo magnetic resonance technique that is sensitive to local perturbations in the magnetic field because of the products of prior hemorrhage, some forms of mineralization, or air. The foci of susceptibility artifact found in our subjects are likely to represent hemosiderin rather than calcium, because calcium deposition is usually a consequence of focal injury, such as ischemia or inflammation. We found no focal signal abnormality in the corresponding regions on $\mathrm{T} 1 \mathrm{w}$ or $\mathrm{T} 2 \mathrm{w}$ scans to suggest that these foci were associated with focal ischemia or inflammation (eg, Figure 1), nor were these foci in brain regions typically affected by ischemic injury in patients with CHD described in prior neuropathologic or MRI 

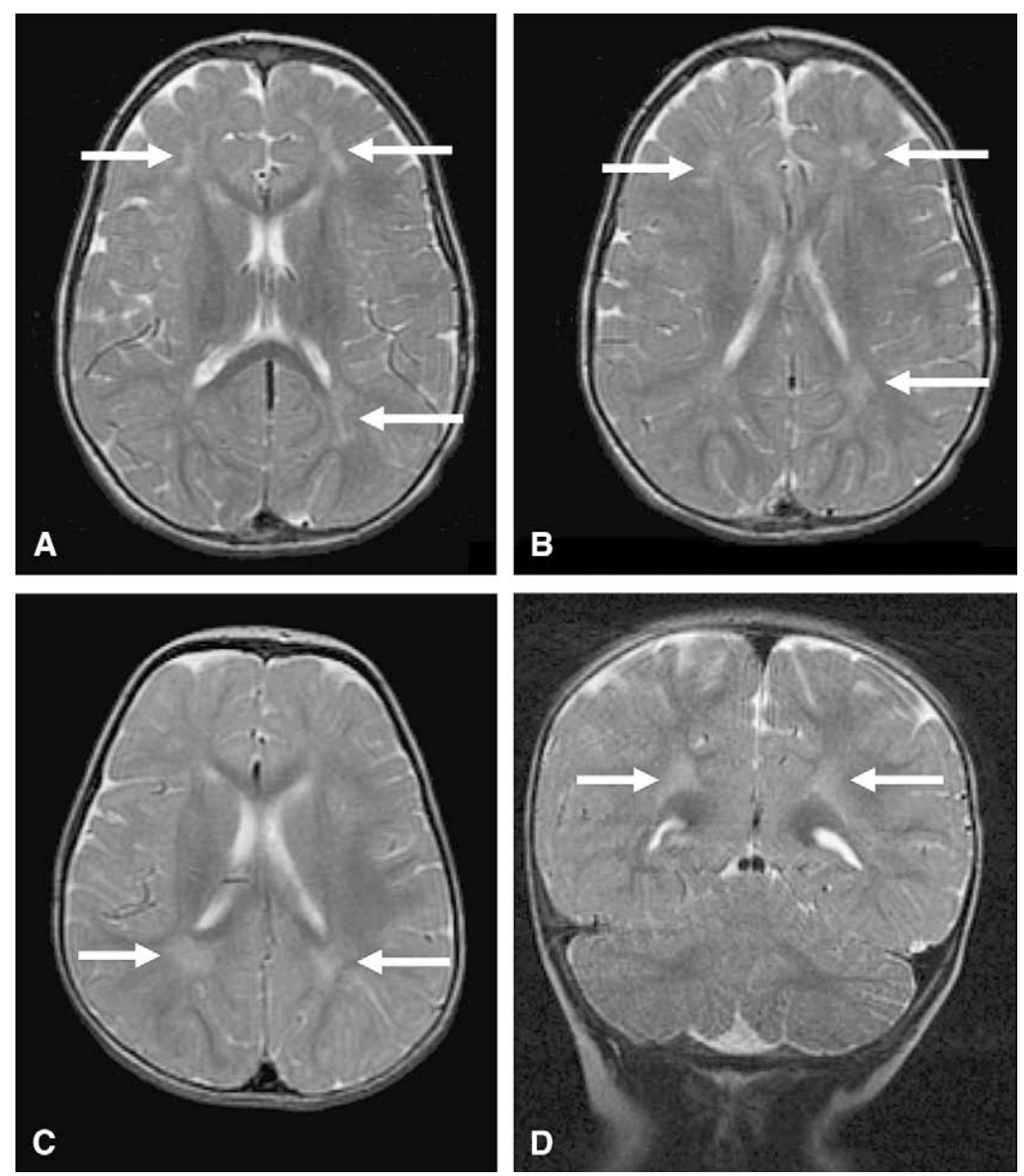

FIGURE 3. PVL in a subject with VSD (A, B) and a subject with TOF (C, D) shown by $T 2 w$ imaging as patchy areas of T2 hyperintensity in the cerebral white matter dorsolateral to the frontal horns and occipital horns of the lateral ventricles (arrows).

studies. ${ }^{10-14,23}$ None of the lesions reported in a previous neuropathologic study from Children's Hospital Boston would be expected to have this type of MRI appearance. ${ }^{10}$ However, this discrepancy could be due in part to differences in the management of patients with CHD, because the neuropathology study was conducted on patients whose cardiac surgery was performed in the period 1985 to 1993. One potential cause for these foci is emboli, possibly related to catheterization, surgical procedures, or bypass support during surgery. Because we did not perform MRI scans before surgical repair, we could not determine whether these foci were present preoperatively. We did not find any association between presence of these foci and administration of antifibrinolytics; thus hematologic management did not seem to be a risk factor. Given that these foci were tiny, any associated parenchymal abnormalities might be better elucidated by using higher resolution conventional and susceptibility-weighted imaging sequences on a high-field (eg, 3T) MRI scanner.

Despite the lack of associated overt parenchymal abnormalities by conventional MRI sequences, the presence of these foci of presumed hemosiderin was associated with significantly lower PDI score at 1 year of age. Their association with developmental outcome supports the notion that these foci may be a marker of brain injury not detected by conventional T1w and T2w MRI sequences. We therefore examined our data to determine if the presence of hemosiderin was associated with perioperative factors known to be associated with brain injury, such as younger age at surgical repair. PVL is found more commonly in children who are repaired as newborns $(54 \%)$ than as infants $(4 \%),{ }^{12}$ and PVL and stroke often occur in the newborn period. ${ }^{13,14}$ We hypothesized that younger age at surgical repair might render immature small cerebral blood vessels more prone to rupture, producing foci of hemosiderin detected by MRI. However, hemosiderin foci were associated with older age at surgery in our subjects, suggesting a greater vulnerability to a different type of brain injury in older infants. A recent study reported that older age at surgery is associated with lower intelligence quotient score at age of school entry, ${ }^{24}$ although this relationship may be confounded by other clinical variables associated with both older age at surgery 


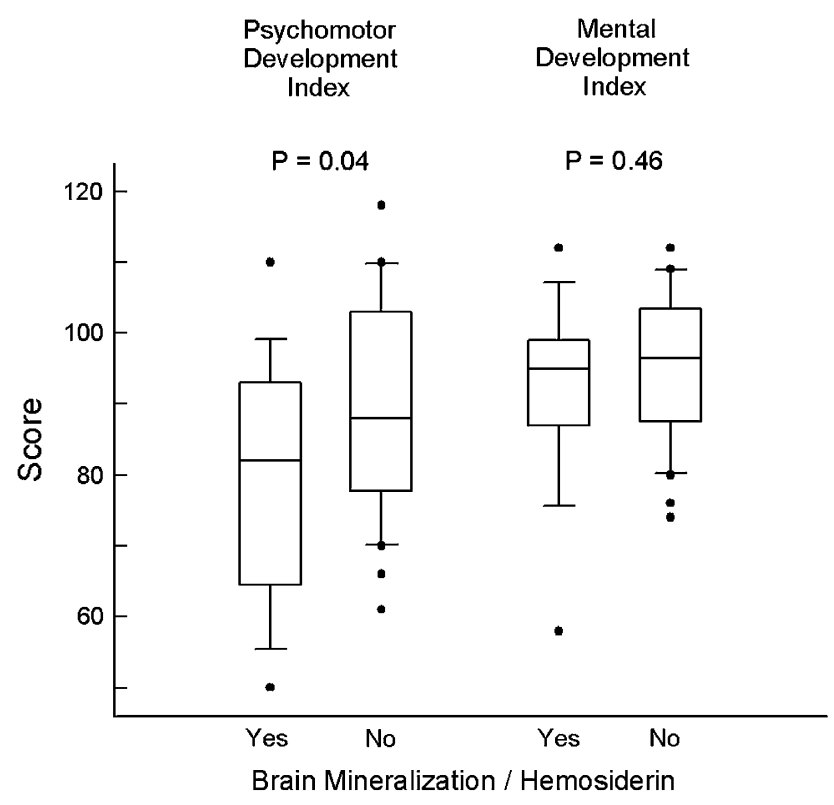

FIGURE 4. Boxplot of PDI and MDI scores for subjects with and without foci of hemosiderin. The limits of the box indicate the 25 th and 75 th percentiles, and the median is marked by the line within the box. The whiskers indicate the 10th and 90th percentiles, and the observations outside of this range are shown as solid circles.

and later neurologic impairments. We found no association of hemosiderin foci with BAS, oxygenation, or intraoperative bypass times, risk factors associated with a higher risk of brain injury in previous studies. ${ }^{12-14}$ Thus, it seems that these foci are a marker of a type of brain injury with different etiologic risk factors than the white matter injury or stroke reported in prior studies of children with CHD.

The low incidence of overt ischemic injury, such as focal stroke or PVL, in our population was an interesting finding. Previous studies have reported that $41 \%$ to $67 \%$ of infants with CHD of all types had ischemic brain injury, such as focal stroke or PVL by brain MRI. ${ }^{11-14}$ In contrast, we found only 1 subject with stroke $(2 \%)$ and 2 subjects with PVL ( $4 \%$ ) by MRI among our 48 subjects. It is possible that subtle ischemic injury may not be detected by brain MRI at 1 year of age, as opposed to the preoperative and early postoperative timing of scans performed in prior studies. ${ }^{11-14,25}$ However, focal stroke of moderate size as reported in previous studies ${ }^{13,14}$ should be readily apparent as areas of encephalomalacia by brain MRI at 1 year of age. Moreover, the 1 subject in our series found to have a stroke had a very small area of focal encephalomalacia, demonstrating the sensitivity of MRI to detect an old small stroke at 1 year of age. It is not known whether the mild PVL reported by other groups would have the same appearance by MRI at 1 year as the PVL in our 2 subjects or whether it would be inapparent by MRI at this later age. A previous report suggested that patients with CHD show resolution of PVL at 4 to 6 months. ${ }^{11}$ However, this observation may be due in part to the relative insensitivity of MRI to detect mild PVL at 4 to 6 months of age, as suggested by the authors. ${ }^{11}$ For infants born prematurely, we have found that MRI scans performed at either term age or 1 year of age or older are best for detection of PVL, because the white matter is of uniform signal intensity, either largely unmyelinated or myelinated at these ages, respectively. This background of uniform signal intensity in the white matter facilitates detection of focal areas of signal abnormality. At 4 to 6 months of age, the cerebral white matter is partially myelinated, ${ }^{26}$ rendering detection of abnormalities more difficult against a background of white matter with intermediate signal intensity. However, the lack of preoperative or early postoperative imaging in our study limits direct comparison of the incidence and severity of white matter injury with previous reports.

Because the incidence of stroke and PVL was low in our study, we could not determine which perioperative clinical factors were associated with these lesions. In particular, we did not find a strong association between BAS and either stroke or PVL. Only 1 of our 17 subjects who underwent BAS had a stroke (incidence of $6 \%$ of those with BAS, or $5 \%$ of all TGA subjects), and none of our subjects with TGA had PVL, compared with a previous report of PVL and stroke combined occurring in $58 \%$ of patients with TGA who underwent BAS. ${ }^{13}$ These discrepant findings raise the question of whether focal stroke and PVL in patients with TGA after BAS may be related to institutional differences in detection or interpretation of PVL by MRI or to differences in management. The strokes reported in prior studies of children with CHD appear to be embolic. ${ }^{11,13,14}$ In our series, only 2 subjects who underwent BAS received heparin; differences between institutions in the occurrence of stroke in subjects with TGA thus are unlikely to be related to use of prophylactic anticoagulation during BAS.

In addition to ischemic injury, previous studies have reported frequent germinal matrix/intraventricular hemorrhage or choroid plexus hemorrhage, subdural hemorrhage, and (rarely) parenchymal hemorrhage occurring in infants with CHD, in both the preoperative and postoperative periods. ${ }^{11,14,22} \mathrm{We}$ acquired a susceptibility-weighted sequence (MPGR) specifically to detect evidence of prior hemorrhage or hemorrhagic stroke, but found neither in our cohort. Evidence of old germinal matrix-intraventricular and parenchymal hemorrhage should be apparent by MPGR sequence 1 year later. Discrepancies in our findings from those in previous reports may reflect differences in management or in the population of subjects with CHD in our study. The lack of specific description of the MRI sequences performed in previous studies precluded us from determining whether differences in detection of hemosiderin foci were related to variation in the acquisition parameters of the susceptibility-weighted sequences. ${ }^{11,12,22}$

Our study should be viewed in light of its limitations. Because we did not perform preoperative scans, we could not 
determine definitively whether hemosiderin foci were present before either catheterization or surgical procedures. Thus we could not determine whether these foci were related to interventions or they might have been present in the preoperative period. In addition, findings in our sample of infants who underwent a single 2-ventricle surgical repair at a single institution may not be generalizable to the broader population of children with CHD.

\section{CONCLUSIONS}

Our data demonstrate a low incidence of focal stroke or PVL, but a new finding of tiny foci of hemosiderin associated with worse developmental outcome at age 1 year among children who underwent 2-ventricle repair in infancy. These data support the notion that neurodevelopmental impairments in children surviving with surgically repaired CHD are due only in part to easily detected neurologic lesions, such as stroke or PVL. Instead, we found that radiologically subtle brain injury was associated with neurodevelopmental impairments in our series, and that this lesion was associated with older age at surgery. This type of brain injury may be better detected with more sensitive or quantitative approaches to MRI data acquisition and analysis and the use of serial imaging studies to determine the timing of injury, as demonstrated in a study using serial diffusion tensor imaging. ${ }^{27}$ The development of a susceptibility-weighted imaging technique that uses a high-spatial resolution 3-dimensional gradient magnetic resonance technique with phase post-processing ${ }^{28}$ may provide an even more sensitive method for detecting small foci of hemosiderin than the conventional 2-dimensional, long TE, T2-weighted gradient recalled echo sequence (with no additional image processing) used in this study. A standardized approach to serial imaging across institutions may help elucidate institutional differences in the incidence, type, and causes of brain injury in children with CHD.

The authors wish to acknowledge the Farb Family Fund for its support.

\section{References}

1. Bellinger D, Jonas R, Rappaport L, Wypij D, Wernovsky G, Kuban K, et al. Developmental and neurologic status of children after heart surgery with hypothermic circulatory arrest or low-flow cardiopulmonary bypass. N Engl J Med. 1995; 332:549-55.

2. Miller G, Tesman J, Ramer J, Baylen B, Myers J. Outcome after open-heart surgery in infants and children. J Child Neurol. 1996;11:49-53.

3. Bellinger DC, Wypij D, Kuban KC, Rappaport LA, Hickey PR, Wernovsky G, et al. Developmental and neurological status of children at 4 years of age after heart surgery with hypothermic circulatory arrest or low-flow cardiopulmonary bypass. Circulation. 1999;100:526-32.

4. Bellinger DC, Bernstein JH, Kirkwood MW, Rappaport LA, Newburger JW. Visual-spatial skills in children after open-heart surgery. J Dev Behav Pediatr. 2003; 24:169-79.

5. Bellinger DC, Wypij D, duDuplessis AJ, Rappaport LA, Jonas RA, Wernovsky G, et al. Neurodevelopmental status at eight years in children with dextro-transposition of the great arteries: the Boston Circulatory Arrest Trial. J Thorac Cardiovasc Surg. 2003;126:1385-96.
6. Karl TR, Hall S, Ford G, Kelly EA, Brizard CP, Mee RB, et al. Arterial switch with full-flow cardiopulmonary bypass and limited circulatory arrest: neurodevelopmental outcome. J Thorac Cardiovasc Surg. 2004;127:213-22.

7. Wernovsky G, Shillingford AJ, Gaynor JW. Central nervous system outcomes in children with complex congenital heart disease. Curr Opin Cardiol. 2005;20:94-9.

8. Majnemer A, Limperopoulos C, Shevell M, Rosenblatt B, Rohlicek C, Tchervenkov C. Long-term neuromotor outcome at school entry of infants with congenital heart defects requiring open-heart surgery. J Pediatr. 2006;148:72-7.

9. Hsia TY, Gruber PJ. Factors influencing neurologic outcome after neonatal cardiopulmonary bypass: what we can and cannot control. Ann Thorac Surg. 2006;81:S2381-8.

10. Kinney HC, Panigrahy A, Newburger JW, Jonas RA, Sleeper LA. Hypoxic-ischemic brain injury in infants with congenital heart disease dying after cardiac surgery. Acta Neuropathol (Berl). 2005;110:563-78.

11. Mahle WT, Tavani F, Zimmerman RA, Nicolson SC, Galli KK, Gaynor JW, et al An MRI study of neurological injury before and after congenital heart surgery. Circulation. 2002;106(12 Suppl. 1):I109-14.

12. Galli KK, Zimmerman RA, Jarvik GP, Wernovsky G, Kuypers MK, Clancy RR, et al. Periventricular leukomalacia is common after neonatal cardiac surgery. J Thorac Cardiovasc Surg. 2004;127:692-704.

13. McQuillen PS, Hamrick SE, Perez MJ, Barkovich AJ, Glidden DV, Karl TR, et al Balloon atrial septostomy is associated with preoperative stroke in neonates with transposition of the great arteries. Circulation. 2006;113:280-5.

14. McQuillen PS, Barkovich AJ, Hamrick SE, Perez M, Ward P, Glidden DV, et al. Temporal and anatomic risk profile of brain injury with neonatal repair of congenital heart defects. Stroke. 2007;38(2 Suppl):736-41.

15. du Plessis AJ, Jonas RA, Wypij D, Hickey PR, Riviello J, Wessel DL, et al Perioperative effects of alpha-stat versus $\mathrm{pH}$-stat strategies for deep hypothermic cardiopulmonary bypass in infants. J Thorac Cardiovasc Surg. 1997;114: 991-1001.

16. Jonas RA, Wypij D, Roth SJ, Bellinger DC, Visconti KJ, du Plessis AJ, et al. The influence of hemodilution on outcome after hypothermic cardiopulmonary bypass: results of a randomized trial in infants. J Thorac Cardiovasc Surg. 2003; 126:1765-74.

17. Newburger JW, Wypij D, Bellinger DC, du Plessis AJ, Kuban KC, Rappaport LA, et al. Length of stay after infant heart surgery is related to cognitive outcome at age 8 years. J Pediatr. 2003;143:67-73.

18. Wypij D, Newburger JW, Rappaport LA, duPlessis AJ, Jonas RA, Wernovsky G et al. The effect of duration of deep hypothermic circulatory arrest in infant heart surgery on late neurodevelopment: the Boston Circulatory Arrest Trial. J Thorac Cardiovasc Surg. 2003;126:1397-403.

19. Tabbutt S, Nord AS, Jarvik GP, Bernbaum J, Wernovsky G, Gerdes M, et al. Neurodevelopmental outcomes after staged palliation for hypoplastic left heart syndrome. Pediatrics. 2008;121:476-83.

20. Newburger JW, Jonas RA, Soul J, Kussman BD, Bellinger DC, Laussen PC, et al Randomized trial of hematocrit $25 \%$ versus $35 \%$ during hypothermic cardiopulmonary bypass in infant heart surgery. $J$ Thorac Cardiovasc Surg. 2008;135: 347-54, 354.e1-4.

21. Maier SE, Gudbjartsson H, Patz S, Hsu L, Lovblad KO, Edelman RR, et al. Line scan diffusion imaging: characterization in healthy subjects and stroke patients. AJR Am J Roentgenol. 1998;171:85-93.

22. Tavani F, Zimmerman RA, Clancy RR, Licht DJ, Mahle WT. Incidental intracranial hemorrhage after uncomplicated birth: MRI before and after neonatal heart surgery. Neuroradiology. 2003;45:253-8.

23. Glauser T, Rorke L, Weinberg P, Clancy R. Acquired neuropathologic lesions associated with the hypoplastic left heart syndrome. Pediatrics. 1990;85:991-1000.

24. Majnemer A, Limperopoulos C, Shevell M, Rohlicek C, Rosenblatt B, Tchervenkov C. Developmental and functional outcomes at school entry in children with congenital heart defects. J Pediatr. 2008;153:55-60.

25. Miller SP, McQuillen PS, Hamrick S, Xu D, Glidden DV, Charlton N, et al. Abnormal brain development in newborns with congenital heart disease. $N$ Engl $J$ Med. 2007:357:1928-38.

26. Barkovich AJ, Kjos BO, Jackson DE Jr, Norman D. Normal maturation of the neonatal and infant brain: MR imaging at $1.5 \mathrm{~T}$. Radiology. 1988;166(1 Pt 1): 173-80.

27. Partridge SC, Vigneron DB, Charlton NN, Berman JI, Henry RG, Mukherjee P, et al. Pyramidal tract maturation after brain injury in newborns with heart disease. Ann Neurol. 2006;59:640-51.

28. Haacke EM, Xu Y, Cheng YC, Reichenbach JR. Susceptibility weighted imaging (SWI). Magn Reson Med. 2004;52:612-8. 